\title{
Modification of the Near-Surface Layers of a Copper Foil under the Action of a Volume Gas Discharge in Air at Atmospheric Pressure
}

\author{
M.A. Shulepov, V.F. Tarasenko, I.M. Goncharenko, N.N. Koval, and I.D. Kostyrya \\ Institute of High-Current Electronics SB RAS, 2/3, Academichesky ave., Tomsk, 634055, Russia \\ Phone: +8(3822) 49-14-43, Fax: +8(3822) 49-24-10, E-mail: MixShlp@yandex.ru
}

\begin{abstract}
We have studied the modification of the near-surface layers of a copper foil under the action of a volume gas discharge, which was generated in air at atmospheric pressure by nanosecond highvoltage pulses of both negative and positive polarity applied between the foil and an electrode with a small radius of curvature. It is established that the surface layer of the discharge-treated copper foil in the central region is cleaned from carbon contaminations, while oxygen atoms penetrate in depth of the foil. The depth of a cleaned layer depends on the polarity of voltage pulses. For the positive voltage polarity on the foil, the cleaning takes place up to a depth exceeding $50 \mathrm{~nm}$, while oxygen penetrates up to a depth of about $25 \mathrm{~nm}$.
\end{abstract}

\section{Introduction}

Electric discharges of different types as well as electron beams are now widely used for the modification of near-surface layers of various materials [1]. It was reported that a high-voltage diffuse discharge at a low gas pressure and a low power could be used for the inactivation of microbiological cultures [2]. As is known [3], a volume discharge can be generated using inhomogeneous electric field in gases at atmospheric pressure. For this purpose, high-voltage $(\sim 100 \mathrm{kV})$ pulses of nanosecond duration are applied to a gasfilled interelectrode gap. A specific feature of such discharges in air at atmospheric pressure is the accompanying X-ray emission [3-5] and the formation of runaway electron beams [5].

In recent years, it was established that a volume discharge can be generated in air at atmospheric pressure in an inhomogeneous electric field for both polarities of the high-voltage pulses applied to an electrode with a small radius of curvature $[6,7]$. Another feature of volume discharges in inhomogeneous electric fields, which are initiated by ultrashort avalanche electron beams (UAEBs), is the possibility of reaching high levels of specific power (up to $800 \mathrm{MW} / \mathrm{cm}^{3}$ [8]) deposited in the discharge. In such regimes, runaway electron beams emitted from the discharge plasma are characterized by the beam current amplitudes reaching tens and hundreds of amperes (behind the foil), while the current pulse duration (defined as the pulse full width at half maximum, FWHM) does not exceed 100 ps [9]. Such discharges in inert gases are also accom- panied by high-intensity vacuum ultraviolet (VUV) emission [10].

Thus, the generation of UAEB-induced volume discharge in gases at high pressures leads to a complex action of the high-density nanosecond discharge plasma (with a specific deposited power of several hundred megawatt per cubic centimeter), UAEBs (with a broad energy spectrum), and radiation in various spectral ranges (including UV and VUV emitted from the discharge plasma) on the anode.

Thus, the generation of UAEB-induced volume discharge in gases at high pressures leads to a complex action of the high-density nanosecond discharge plasma (with a specific deposited power of several hundred megawatt per cubic centimeter), UAEBs (with a broad energy spectrum), and radiation in various spectral ranges (including UV and VUV emitted from the discharge plasma) on the anode.

This Letter presets the results of an experimental investigation of the modified near-surface layers of a copper foil upon the action of plasma of a UAEBinduced volume discharge in air at atmospheric pressure.

\section{Setup and methods of experiment}

The experiments were performed using a discharge chamber, which is schematically depicted in Fig. 1.

A distance from a flat copper foil to a tubular electrode could be varied within $8-16 \mathrm{~mm}$. The interelectrode voltage was supplied from an oscillator of the RADAN-220 type, which generated voltage pulses with an amplitude of $\sim 230 \mathrm{kV}$ (in the open-circuit regime), a FWHM of $\sim 2$ ns (on a matched load), and a leading front width of $\sim 0.5 \mathrm{~ns}$ [11]. It was possible to change the polarity of generated voltage pulses applied to the potential electrode. The discharge current was measured using a shunt composed of resistor chips connected between the foil anode and the discharge chamber housing. The results of measurements showed that the current pulse amplitude for both polarities of the applied voltage pulse was $\sim 3 \mathrm{kA}$ and the total duration of the discharge current pulse was $\sim 30 \mathrm{~ns}$ (the first half- period of the discharge current pulse had a duration of $\sim 8 \mathrm{~ns}$ ). With the negative polarity of a voltage pulse applied to the electrode with a small curvature radius, the gas-filled diode generated a beam of runaway electrons. Under these conditions, 


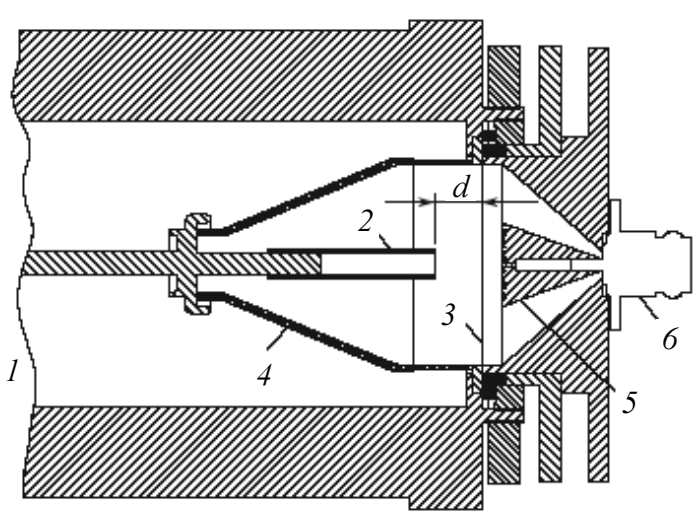

$a$

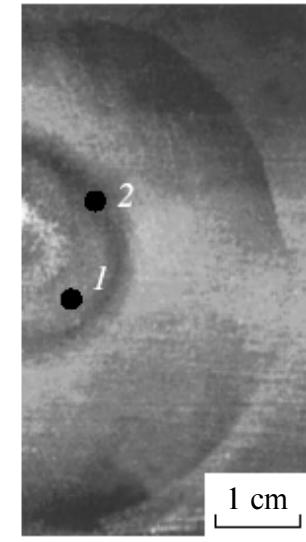

$b$

Fig. 1. Schematic diagram of the gas-filled diode and collector assembly: 1 - connection to a high-voltage pulse generator; 2 - cathode; 3 - foil anode; 4 - insulator; 5 - cone-shaped collector; 6 - connecting socket $(a)$; photograph of a copper foil (half) after exposure to the volume discharge plasma and runaway electron beam at a gap width of $d=11 \mathrm{~mm}$ between the foil and the cathode; points $l$ and 2 indicate the sitesof AES analysis $(b)$

the electron beam current was measured using a coneshaped collector (see Fig. 1), while the copper foil was replaced by a $45-\mu$ m-thick AlBe foil. For electrons with energies above $70 \mathrm{keV}$ and a current pulse width (FWHM) of $\sim 0.1 \mathrm{~ns}$ (which corresponded to the given collector resolution), the current pulse amplitude was $\sim 10 \mathrm{~A}$. The electron energy distribution measured in an analogous diode filled with air at atmospheric pressure was reported elsewhere $[9,12]$. The beam energy spectrum in a diode used in the present study contained electrons with energies from 40 to $180 \mathrm{keV}$. It should be noted that electrons with the energies below $40 \mathrm{keV}$ cannot be detected by the method employed in $[9,12]$, although calculations $[13,14]$ show that a considerable fraction of particles with such energies must be formed in the discharge plasma.

The samples of copper foil were irradiated in a periodic pulse regime at a pulse repetition rate of $1 \mathrm{~Hz}$. The irradiated samples were studied by Auger electron spectroscopy (AES) so as to reveal changes in the chemical composition of a near-surface layer of the material.

Figure $1, b$ shows a photograph of the surface of a 100 - $\mu \mathrm{m}$-thick copper foil upon the exposure to 300 discharge pulses. As can be seen, there are three characteristic regions. The first circular region with a diameter of $\sim 16 \mathrm{~mm}$ corresponds to the size of highdensity volume discharge plasma contacting with the foil (point 1). A narrow bright ring observed inside this region results from the contraction of discharge during some pulses generated at an interelectrode gap width of $11 \mathrm{~mm}$. When the gap width was increased to 14-16 mm, the discharge always exhibited a volume character, but the discharge current and the electron beam current densities exhibited a decrease. The second region appears as a dark ring with a width of $\sim 2 \mathrm{~mm}$ (Fig. 1, $b$, point 2). The third region represents a peripheral zone, where the discharge current and the electron beam current densities (at a positive voltage polarity on the foil) were small.
Figure 2 shows the results of investigation of the results of discharge-induced modification of the nearsurface layers of a copper foil.
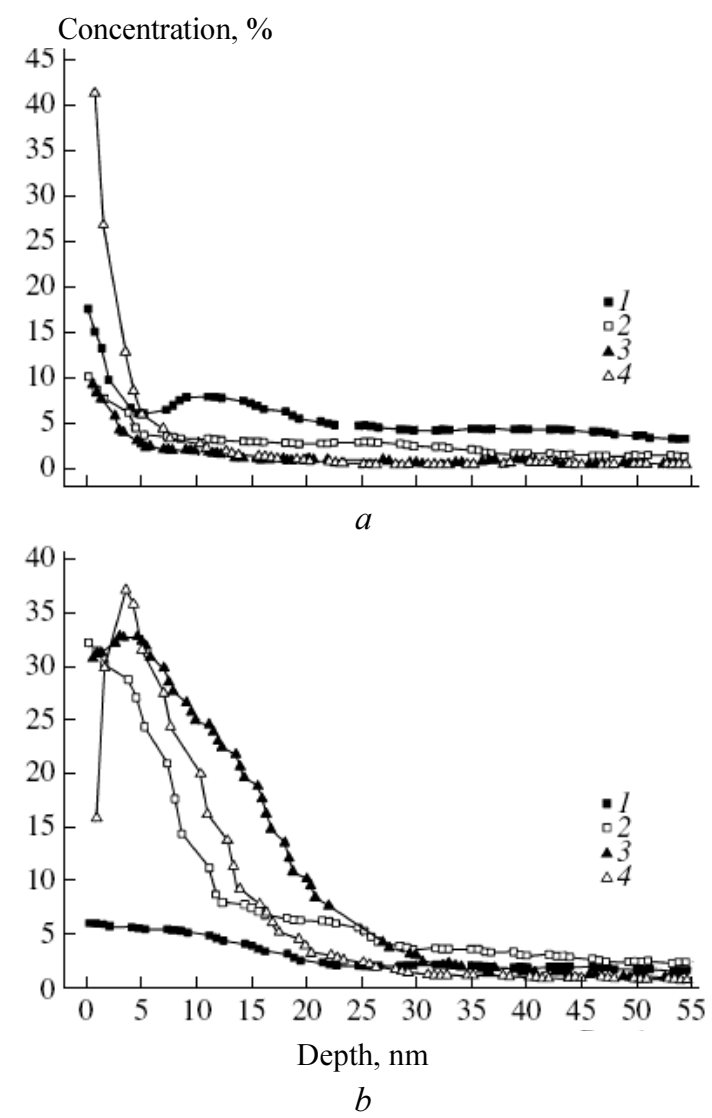

Fig. 2. Depth-concentration profiles of carbon $(a)$ and oxygen $(b)$ in the near-surface layers of a copper foil before (initial sample) (1) and after exposure (2-4) to a UAEBinduced volume discharge in air at atmospheric pressure. The AES depth profiling was performed at point $l$ (curves 2 and 3 ) and point 2 (curves 4 ) indicated in Fig. 1, $b$; the foil was connected as the cathode (curve 2) and as the anode (curves 3,4) of the gas-filled diode 
The modification was characterized by the profiles of carbon (Fig. 2, a) and oxygen (Fig. 2,b) concentrations in depth of the samples, which were obtained using the AES depth profiling technique. The chemical composition was analyzed at two points of each sample (Fig. 1,b), which corresponded to the regions of maximum plasma action (point 1 ) and the maximum change in the color of the sample surface (point 2). As can be seen, the exposure to the UAEBinduced volume discharge in air at atmospheric pressure leads to cleaning of the copper surface from carbon contaminations (point 1 ) and to an increase in the oxygen concentration in the near-surface layers (points 1 and 2). The most pronounced effect was observed for the negative polarity of a voltage pulses applied to the electrode with a small curvature radius (Fig. 2, curves 3). In this case, the foil was under the action of both discharge plasma and a beam of runaway electrons with various energies (ranging from several units to hundreds of kiloelectronvolts). Under such conditions, the cleaning from carbon in comparison to the initial sample (Fig. 2, $a$, curve 1 ) was observed at a depth exceeding $50 \mathrm{~nm}$. The oxygen concentration was also significantly increased in comparison to that in the initial sample (Fig. 2, b, curve 1 ) at a depth of up to about $25 \mathrm{~nm}$.

The AES data indicate that the oxygen concentration in the near-surface layer is substantially increased both at point 1 and at point 2 , while the concentrations of carbon in a $\sim 10$-nm-thick layer at these points are significantly different. The maximum concentration of carbon at point 2 (Fig. 2, a, curve 4) after the discharge treatment is about four times that at point 1 (curve 2). Moreover, the concentration of carbon at point 2 is more than twice as large as that in the initial sample. This increase in the concentration of carbon can be related to inhomogeneity of the discharge current distribution over the anode surface. A dense discharge region is confined within the dark ring (on which point 2 is located), which can also be seen on photographs of the discharge glow.

It should be noted that a discharge with the positive polarity of voltage pulses on the electrode with a small radius of curvature is also accompanied by the generation of runaway electrons and X-ray quanta, which leads to the formation of a volume discharge in the inhomogeneous electric field even without an additional preionization. However, the energy of runaway electrons generated in this regime does not exceed 10-20 keV, which complicates their monitoring. The X-ray emission from a gas-filled diode could be detected only using a tungsten anode with a small radius of curvature and an interelectrode gap width reduced to $\sim 0.5 \mathrm{~mm}$. X-ray emission from an interelectrode gap under analogous conditions was previously also observed in [4].

It should be noted that, while the conditions of UAEB-induced volume discharge formation admit broad variation of the voltage pulse duration and am- plitude and the cathode design [6, 8-14], the generation of a runaway electron beam requires the optimization of a number of parameters [9]. The present study was not aimed at studying the surface modification of copper under the conditions of runaway electron beam formation with maximum amplitude. For this purpose, it would be necessary to optimize the voltage pulse amplitude and leading front duration, the cathode material, the interelectrode gap width, and the general gas-filled diode and cathode design.

\section{Conclusion}

Thus, the results of our investigation showed that the exposure of a copper foil in a UAEB-induced volume discharge in air at atmospheric pressure is accompanied by the cleaning and modification of a nearsurface layer of the foil. The anode is subjected to a complex action of the high-density nanosecond discharge plasma (with a specific deposited power of several hundred megawatt per cubic centimeter) and radiation in various spectral ranges (including UV and VUV) on the anode. We have also observed the influence of a UAEB on the modification of the copper foil surface. The electron beam was detected behind an AlBe foil for the negative polarity of a voltage pulse applied to the electrode with a small curvature radius.

The UAEB-induced volume discharge can be readily obtained in various gases in a broad range of pressures; at a reduced pressure, the electron beam current density in helium can reach up to $2 \mathrm{kA} / \mathrm{cm} 2$ [15]. Therefore, the UAEB-induced volume discharge can be used for the modification of metal surfaces in various technological processes, while systems with special anode design can also be used for the surface modification of dielectrics.

\section{Acknowledgments}

The authors are grateful to A.B. Markov for fruitful discussions and to I.A. Shulepov for testing samples.

\section{References}

[1] A.N. Didenko, Yu.P. Sharkeev, I.V. Kozlov et al., Long-Range Effects in Ion-Implanted Metallic Materials, Tomsk, NTL, 2004.

[2] S.N. Buranov, S.V. Voevodin, I.A. Voevodina et al., Teplofiz. Vys. Temp. 43, 367 (2005).

[3] L.V. Tarasova and L.N. Khudyakova, Zh. Tekh. Fiz. 39, 1530 (1969) [Sov. Phys. Tech. Phys. 14, 1148 (1969)].

[4] Yu.L. Stankevich and V.G. Kalinin, Dokl. Akad. Nauk SSSR 177 (1), 72 (1967) [Sov. Phys. Dokl. 12,1148 (1967)].

[5] L.V. Tarasova, L.N. Khudyakova, T.V. Loiko, and V.A. Tsukerman, Zh. Tekh. Fiz. 44, 564 (1974) [Sov. Phys. Tech. Phys. 19, 351 (1974)].

[6] I.D. Kostyrya and V.F. Tarasenko, Izv. Vyssh. Uchebn. Zaved., Fiz. 47 (12), 85 (2004). 
[7] I.D. Kostyrya, V.M. Orlovskiњ, and V.F.Tarasenko, et al., Pis'ma Zh. Tekh. Fiz. 31 (11), 19 (2005) [Tech. Phys. Lett. 31, 451 (2005)].

[8] S.B. Alekseev, V.P. Gubanov, and I.D. Kostyrya, et al., Kvantovaya Elektron. 34, 1007 (Moscow, 2004).

[9] V.F. Tarasenko, V.G. Shpak, S.A. Shunailov, and I.D. Kostyrya, Laser Part. Beams 23, 545 (2005).

[10] E.Kh. Baksht, D.V. Rybka, M.I. Lomaev, and V.F. Tarasenko, Kvantovaya Elektron. 36, 576 (Moscow, 2006).
[11] M.I. Yalandin and V.G. Shpak, Prib. Tekh. Eksp., No. 3, 5 (2001)

[12] V.F. Tarasenko, I.D. Kostyrya, V.K. Petin, and S.V. Shlyakhtun, Zh. Tekh. Fiz. 76 (12), 37 (2006) [Tech. Phys. 51, 37 (2006)].

[13] I.D. Kostyrya, V.F. Tarasenko, A.N. Tkachev, and S.I. Yakovlenko, Pis'ma Zh. Tekh. Fiz. 33 (7), 79 (2007) [Tech. Phys. Lett. 33, 309 (2006)].

[14] V.B. Bratchikov, K.A. Gagarinov, and I.D. Kostyrya, et al., Zh. Tekh. Fiz. 77 (7), 34 (2007) [Tech. Phys. 52, 856 (2007)]. 